\title{
Study on Emergency Management System of Dangerous Chemicals Accidents based on the Dynamic Risk
}

\author{
Xuanya Liu ${ }^{1}$, Jingjing $\mathrm{Li}^{1}$, Ning $\mathrm{Zhou}^{2}$ \\ ${ }^{1}$ Tianjin Fire Research Institute of MPS, Tianjin 300381, China \\ ${ }^{2}$ Changzhou University, Changzhou, Jiangsu 213016, China

\section{基于动态风险的危化品灾害事故应急管理体系研究 \\ 刘晅亚 $^{1}$ 李晶晶 $^{1}$ 周宁 $^{2}$ \\ 1 公安部天津消防研究所, 天津 300381 , 中国 \\ 2 常州大学, 江苏, 常州 213016, 中国}

\begin{abstract}
According to the characteristics and evolution law of dangerous chemicals accidents, based on disaster accident emergency handling requirements, the research on dynamic risk analysis and prediction technology system of dangerous chemicals accidents were carried out based on real-time dynamic information of accidents. In order to identify the risk factors, the network model of chain accidents causes was set up. And the disaster accident risk prevention and emergency management mode was built based on the dynamic risk monitoring and early warning, which implement the dynamic information integration of risk real-time prediction and emergency management. By using the emergency management system, it can effectively improve the disaster accidents emergency handling skills and abilities.
\end{abstract}

Keywords: dynamic risk; dangerous chemical accidents; chain accident cause; emergency management

\section{摘要}

针对危化品灾害事故特点及其演化发展规律, 基于危 化品灾害事故应急处置需求, 开展基于危化品灾害事 故实时动态信息的态势分析与动态风险预测的技术 体系研究, 构建危化品连锁灾害事故致因网络模型, 建立基于动态风险监测预警的危化品灾害事故风险 防范与消防应急管理模式, 实现对危化品动态灾害事
故风险一体化实时预测与应急管理, 可有效提高针对 危化品灾害事故应急处置技术水平和能力。

关键词: 动态风险; 危化品灾害事故; 连锁事故致因; 应急管理

1. 引言

随着经济的快速发展, 我国对化工产品和能源的需求 急速增长, 相应的易燃易爆危化品的应用也日益增 多。易燃易爆危化品生产、储存、运输及使用等环节 极易发生火灾、爆炸及泄漏扩散等恶性灾害事故, 并 具有多米诺效应显著、事故波及范围广等特点。危化 品灾害事故科学高效处置与事故管理是防范、控制、 减轻事故后果影响, 避免事故进一步发展的关键环 节。针对危化品灾害事故风险, 国内外学者在风险评 估、监管、应急救援等方面开展了大量研究, 如国外 引入贝叶斯网络理论对系统安全屏障进行动态分析, 应用混沌理论分析动态风险变化, 并就危险化学品配 送路线和配送中心建设位置优化等内容开展研究。国 内针对危化品动态风险分析相关研究较为缺乏, 近年 来开始涉及动态概率分析模型、基于模糊数学的概率 动态风险评估方法等研究, 初步建立了动态风险分析 方法的基本思路和理论模型。如柴保身阐述危化品仓 储业现状并预测发展趋势 ${ }^{[1]}$ 、郁振山从消防救援角度

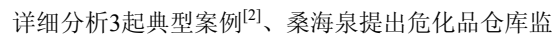
控系统的设计方案并开发软件 ${ }^{[3]}$ 、孙伟等应用信息化 技术研发危化品服务平台 ${ }^{[4]}$ 、张超等建立基于事故后 果评价的危化品风险评估模型 ${ }^{[5]}$ 、Farid Kadri等人基 于多米诺效应对化工企业火灾、爆炸事故风险进行了 研究 ${ }^{[6]}$ 。但在危化品事故影响因素分析、事故动态风 
Risk Analysis and Crisis Response in Big Data Era (RAC-16)

险预测方面, 目前研究尚不深入, 未考虑火灾爆炸连 锁事故特点。

危化品灾害事故事前预警和事故应急联控, 及常 态下各部门、各地区协同配合与信息共享对于危化品 常态安全管理和事后应急快速处置至关重要。危化品 常态安全管理中, 合理的风险等级评估可对应急资源 布置、监管力度分配等提供依据。如中国安全生产科 学研究院开展危化品企业安全生产风险评估分级研 究 ${ }^{[7]}$, 提出固有风险和动态风险相结合的危化品安全 生产风险评估方法, 从企业自身水平(危化品物质量、 工艺水平、安全监控、环境等)和企业安全管理绩效 抽取共性和个性安全生产指标, 采用专家打分和层次 分析法评估企业风险, 并按照风险优先原则实现差别 监管。上海大学赵来军等在危化品运输区域性监管和 联控机制方面 ${ }^{[8]}$, 分析危化品安全监管网络中法律 法规、监管部门信息共享、合作协调机制等方面问题, 提出建立监管部门常态化合作协调机制、无缝化城市 安全监管平台以及跨区域合作监管机制的对策。通过 研究苏浙沪地危化品运输的无缝化区域联控机制, 指 出5类监管缝隙, 用无缝隙化组织理论设计出苏浙沪 危化品跨省运输监管区域联控架构。总体而言, 危化 品运输区域联控机制及平台研究还处于起步阶段, 学 者研究甚少, 是危化品运输从单一模式转向综合监管 模式的必经阶段, 也将会对危化品监管模式产生变革 性影响。

危化品事故处置是一个多部门合作、分工明确、 综合协调的体系, 危化品全生命周期的信息资源共享 与及时传递成为其中需解决的重要问题。信息化技术 为解决此类问题提供有效手段, 但目前已有研究主要 应用 GIS、GPS、RS、RFID、ITS、GPRS、GSM 等 技术建立危化品运输安全监管系统, 主要用于跟踪、 监控车辆信息, 但较少涉及数据分析、事故预测预警、 突发事件方案机制的研究。近几年虽然相关学者也开 始扩展事故模拟、疏散决策等危化品信息化功能, 但 应用范围局限于某一企业或某一行业领域, 尚未形成 统一的技术平台。

本文从典型事故案例入手, 分析事故连锁演化过 程, 提取事故致因因素, 采用 DEMATEL 方法探究 因素逻辑关系, 构建网络模型为连锁事故风险预控提 供指导, 为应急灾情判断、预警、决策分析提供数据 支持; 并基于动态风险分析与信息化技术，构建了危 化品综合信息监管与事故灾害应急管理技术体系, 可 为危化品全生命周期的风险管理与事故应急处置提 供信息技术支持。

\section{2. 危险化学品事故特征及案例剖析}

危化品事故类型主要包括: (1) 火灾, 大多数危化品 燃烧时会产生有毒气体或烟雾, 因此中毒和窒息是人 员伤亡主要原因; (2) 爆炸, 危化品发生化学反应的 爆炸事故或液化气体和压缩气体的物理爆炸事故;

（3）泄漏, 气体或液体危化品若发生一定规模的泄 漏, 泄漏扩散气体或液体遇到点火源容易演变为火 灾、爆炸及其连锁事故, 造成严重的财产损失或环境 污染等。

危化品灾害事故具有以下特征：（1）全周期性, 即在危化品生产、使用、储存、经营、运输、废弃等 各个环节, 在危化品产生至消亡的整个过程都可有能 发生事故。(2) 复杂性, 即危化品物质种类复杂性, 其导致火灾爆炸事故物料种类繁多、物理化学性质各 异; 事故发生原因复杂, 即人的不安全行为、设备隐 患缺陷、环境不安全状态、管理失误等, 任何一个方 面缺陷都可能引发危化品事故发生。(3) 突发性, 危 险化学品物质特性决定其对温度、压力等环境参数要 求严格, 危化品相互之间也可能会发生化学反应, 破 坏稳定性, 从而引发事故的发生从而导致事故发生, 其事故发生具有一定的突发性和非可控性。(4) 事故 后果扩散性, 危险化学品燃烧、爆炸、泄漏事故容易 造成化学品扩散、有毒有害物质泄漏量大, 事故波及 范围广、事故危害影响大。事故发生过程中易引发连 锁灾害事故, 生产装置、储运设施、施工机具的大型 化使危险化学品疗存量上升, 其风险和危害程度增 大, 危化品运行条件苛刻, 任何一个失误可能导致灾 难性后果, 引发连锁事故反应。

2015 年 8 月 12 日, 位于天津市滨海新区天津港 的瑞海国际物流有限公司危险品堆场发生特别重大 火灾爆炸事故, 造成 165 人遇难、 798 人受伤、 54 万平方米区域建筑物严重受损、直接经济损失 68.66 亿元。事故期间共发生 2 次大爆炸、 6 处火灾 ${ }^{[9]}$ 。事 故起因是集装箱内硝化棉湿润剂散失, 出现局部干 燥, 在高温环境作用下加速分解反应, 产生大量热量 并不断积聚, 导致硝化棉温度持续升高, 达到其自燃 温度发生自燃, 并引起相邻集装箱内的硝化棉和其他 危险化学品长时间大面积燃烧, 最终导致堆放于运抵 区的硝酸铵等危险化学品发生爆炸。基于事故连锁理 论, 从事故链角度剖析事故发展过程如图 1 所示。 
Risk Analysis and Crisis Response in Big Data Era (RAC-16)

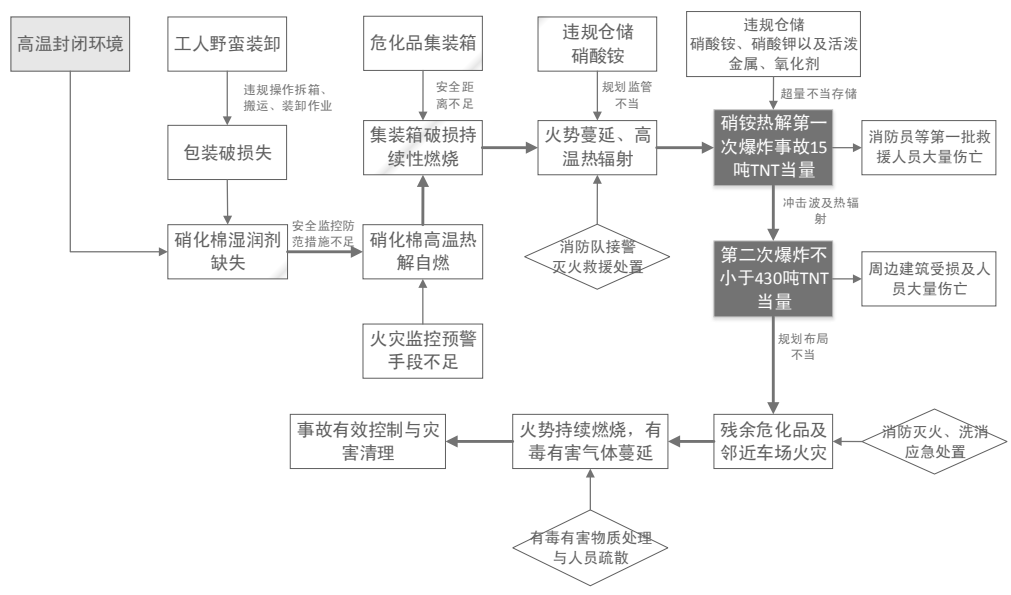

图 1.“ 8.12 天津瑞海特大火灾爆炸事故链

在图 1 事故链中, 重点存在以下几个关键点:

（1）初始火灾发生: 危化品物流堆场、仓库违 规改建、危化品集装箱堆垛仓库管理员专业知识与安 全意识匮乏 $\rightarrow$ 野蛮装卸、拆箱 $\rightarrow$ 包装破损 $\rightarrow$ 硝化棉湿 润剂缺失 $\rightarrow$ 硝化棉高温热解自燃。

（2）火灾至初次爆炸演化：初始火灾发生时无 有效处置 $\rightarrow$ 火灾热量积累 $\rightarrow$ 硝酸铵集装箱爆炸。

（3）连锁火灾爆炸演化：违规超量仓储危化品 $\rightarrow$ 硝酸铵、硝酸钾、硝酸钙、甲醇钠、金属镁、金属 铻、硅钻、硫化钠等氧化剂、易燃固体和腐蚀品的集 装箱热辐射、冲击波引爆 $\rightarrow$ 形成爆炸混合物并发生连 锁爆炸 $\rightarrow$ 爆炸飞火扩大燃烧范围。

(4) 火灾控制与抑制: 消防应急人员采取多项 措施降温、控火、扑灭明火, 排查、检测、洗消、清 运、登记、回炉等程序, 科学慎重清理危险化学品,
在事故中心区周围构筑 1 米高围埝, 封堵 4 处排海口、 3 处地表水沟渠和 12 处雨污排水管道, 把污水封闭 在事故中心区内。同时, 对事故中心区及周边大气、 水、土壤、海洋环境实行 24 小时不间断监测, 采取 针对性防范处置措施, 防止环境污染扩大。

\section{3. 危化品堆场、仓库燃爆连锁事故致因因素提取}

以典型事故调查报告、GB 15603-1995《常用危险化 学品财存通则》、国务院 591 号令《危险化学品安全 管理条例》为参考资料, 结合危险化学品物质特性, 构建危化品仓库燃爆事故致因因素集如图 2 所示。事 故发生的直接原因往往是物的不安全状态和人的不 安全行为, 间接原因是管理问题。从它存环境和尖存 方式提取影响物质状态致因, 危化品管理角度提取管

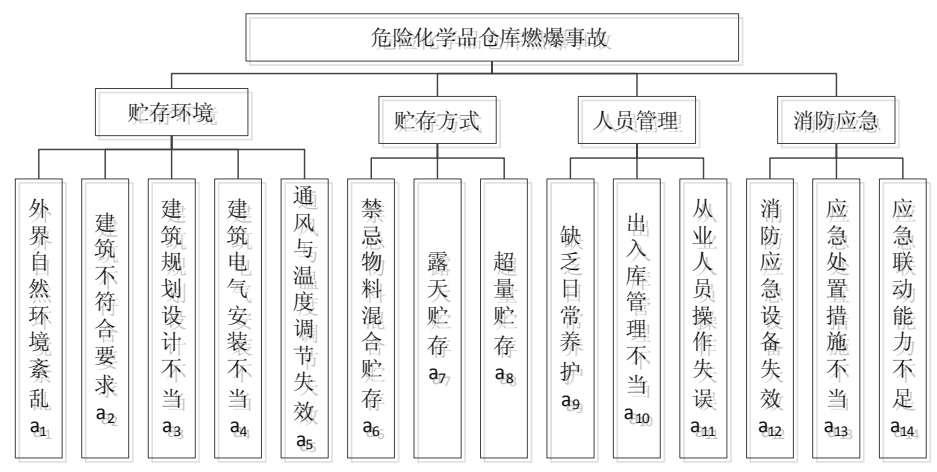

图 2. 危险化学品堆场、仓库爆燃事故致因因素 
Risk Analysis and Crisis Response in Big Data Era (RAC-16)

理致因。考虑到火灾爆炸连锁发展问题, 添加消防应 急作为一级致因类别。

（1）贮存环境包括外界自然环境和建筑咜存场 所。外界自然环境紊乱, 如遭受雷击或周围环境烟图 飞火等可能成为点火源。建筑物耐火等级、防火间距、 选址等影响火灾爆炸事故的抵御能力。建筑场所安装 防爆电气设施、通风调节装置等, 有助于消除点火源。

（2）危险化学品咜存必须遵照国家法律、法规 及相关部门标准, 根据物质特性分区、分类、分库咜 存, 避免禁忌物料接触反应导致燃爆。

（3）危化品入库时严格检查物质质量、包装情 况，它存期间采取适当养护措施，以避免物质品质变 化发生反应。对于危化品的装卸、搬运操作及进出车 辆严格管理, 有助于杜绝因摔、碰、撞击等过程产生 火花和汽车排气管火星。否则会引入点火源产生火 灾。

（4）及时有效消防应急措施, 能够在火灾事故 早起控制事态发展, 避免爆炸连锁事故发生和事故规 模扩大。由于不同危化品及在不同情况下发生火灾时 其火灾方法差异较大, 若处置不当反而会使事故进一 步扩大

\section{4. 危化品堆场、仓库燃爆事故致因分析}

\section{1. 方法介绍}

决策试验与评价实验室法(decision making trial and evaluation laboratory, DEMATEL)是以矩阵和图论作 为工具的系统因素分析方法 ${ }^{[10]}$, 目前已在教育、海运、 医疗、机械等领域运用。该方法通过建立各影响因素 的直接影响关系矩阵 D, 通过规范化处理进行矩阵计 算, 量化影响程度并以因果图形式直观展现因素相互 依赖关系 ${ }^{[11-12]}$, 主要步骤为: (1) 建立直接影响关系 矩阵 D; (2) 计算初始影响关系矩阵 X; (3) 计算综合 影响矩阵 $\mathrm{T}$; (4) 评估因素影响程度、原因度、中心 度; (5) 分析因素相互依赖关系。

$$
\begin{gathered}
X=\frac{D}{\max _{1 \leq i \leq j} \sum_{j=1}^{n} d_{i j}} \\
T=X(I-X)^{-1} \\
r=\left[r_{i}\right]_{n \times 1}=\left[\sum_{j=1}^{n} t_{i j}\right]_{n \times 1} \\
c=\left[c_{j}\right]_{1 \times n}=\left[\sum_{i=1}^{n} t_{i j}\right]_{1 \times n}
\end{gathered}
$$

式中, ri 表示第 $\mathrm{i}$ 个因素对其他因素的直接影响 和间接影响综合程度; cj 表示第 $\mathrm{j}$ 个因素受其他因素
的直接影响和间接影响综合程度。中心度 ri+ci 表示 因素 i 在系统中的重要性程度; 原因度 ri-ci 表示因素 $\mathrm{i}$ 对其他因素的逻辑关系重要性程度。

\section{2. 事故致因逻辑关系分析}

由于危化品仓储然爆事故较为复杂, 因素间存在不同 程度的相互影响关系。以所提取的致因因素为基础, 采用 DEMATEL 方法对上述 14 个因素进行逻辑关系 辨识。依托专家经验分别对致因因素间影响关系按照 程度高低进行赋值打分。直接影响关系矩阵 dij 以 $0-4$ 为因素影响关系评判标准, 表示因素之间没有影响、 低度影响、中度影响、高度影响、极高度影响。通过 对矩阵进行规范化处理等确定因素的中心度和原因 度如表 1 所示, 绘制因素因果图如图 3 所示。

\begin{tabular}{ccccc}
\multicolumn{5}{c}{ 表 1. 危化品仓储爆燃事故致因分析结果 } \\
\hline 致因因素 & 影响度 & 被影响度 & 中心度 & 原因度 \\
\hline $\mathrm{a}_{1}$ & 0.9911 & 0.1429 & 1.1339 & 0.8482 \\
$\mathrm{a} 2$ & 1.2536 & 0.0000 & 1.2536 & 1.2536 \\
$\mathrm{a} 3$ & 0.7334 & 0.0000 & 0.7334 & 0.7334 \\
$\mathrm{a} 4$ & 0.0000 & 0.1662 & 0.1662 & -0.1662 \\
$\mathrm{a} 5$ & 0.0000 & 0.3294 & 0.3294 & -0.3294 \\
$\mathrm{a} 6$ & 0.2857 & 0.9758 & 1.2616 & -0.6901 \\
$\mathrm{a} 7$ & 0.0000 & 0.3324 & 0.3324 & -0.3324 \\
$\mathrm{a} 8$ & 0.3061 & 1.1416 & 1.4477 & -0.8355 \\
$\mathrm{a} 9$ & 0.1837 & 0.1662 & 0.3499 & 0.0175 \\
$\mathrm{a} 10$ & 0.8834 & 0.3324 & 1.2157 & 0.5510 \\
$\mathrm{a} 11$ & 1.9375 & 0.1633 & 2.1008 & 1.7743 \\
$\mathrm{a} 12$ & 0.0000 & 1.0610 & 1.0610 & -1.0610 \\
$\mathrm{a} 13$ & 0.0000 & 1.4575 & 1.4575 & -1.4575 \\
$\mathrm{a} 14$ & 0.1429 & 0.4488 & 0.5917 & -0.3059 \\
\hline
\end{tabular}

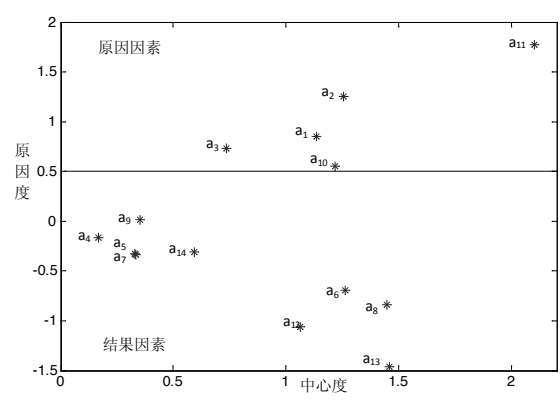

图 3. 致因因素因果图

分析计算结果可知, 危化品仓储区域爆燃事故的 原因因素集中在外界环境、存储建筑物不符合要求、 建筑物规划设计不当、操作人员失误、出入库管理不 当。中心度较大的因素有禁忌物品混合存储、超量存 储、操作人员失误、应急处置措施不当等, 在事故预 防中应重点关注。其中操作人员失误属于原因因素且 中心度较大, 为大部分事故的本质根源。 
Risk Analysis and Crisis Response in Big Data Era (RAC-16)

\section{3. 致因网络模型}

采用解释结构模型探讨各层次间因素递减关系, 建立 危化品仓储燃爆事故的风险致因层次网络, 可为危化 品企业日常管理和运行提供重点参考对象。以 DEMATEL 建立的直接影响矩阵为基础, 选取阈值建 立目标对象的可达矩阵, 分别求解先行集合和可达集 合以完成致因因素的级别分配, 得到因素网络图。相 关学者已对 DEMATEL 和 ISM 方法集合的理论依据、 思路和方法进行详细阐述 ${ }^{[1]}$, 并且在交通领域进行应 用 ${ }^{[12-13]}$, 证明其有良好的结合性和应用价值。因此遵 循上述思路建立致因网络模型如图 4 所示。

第一层为表层致因, 中间层为过渡致因, 底层为 本质致因。表层致因多为事故直接影响因素, 底层致 因通过多种途径对其他因素产生直接影响和间接影 响。因素间存在同层级和跨层级的复杂关联。

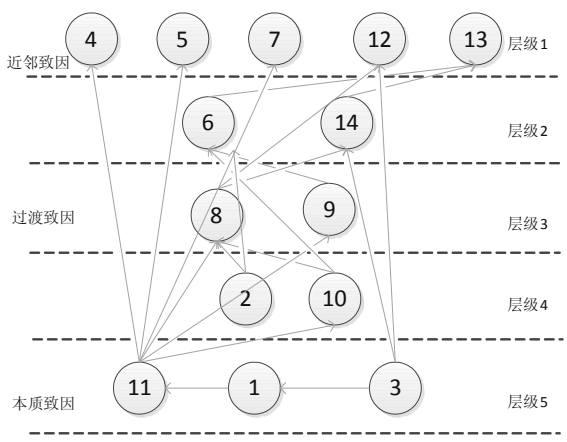

图 4. 致因网络层次结构

\section{5. 基于动态风险的危化品灾害事故应急管理模式}

针对危化品事故发生原因, 以及事故连锁灾害事故的 主要影响因素, 进一步加强对危化品动态风险监管与 事故预测分析, 提高针对危化品事故风险管理与应急 处置技术水平, 对防范危化品灾害事故发生、降低危 化品事故危害影响具有十分重要的意义和作用。 而当前危化品仓储运输呈现大容量、多品种、频繁装 卸储运等特征, 储量远远超越重大危险源临界量, 使 得危险化学品总体事故风险较高, 现有的危化品安全 监管体系虽对有效控制和预防危险化学品的危害起 到了一定的积极作用, 但已不能适应目前危化品事故 应急处置的需要, 应急管理系统由于人力和物力的限 制, 事故应急监测、处置与风险管理水平相对较低。 对于危化品在生产、使用的全生命周期内, 其火灾、
爆炸灾害事故风险与危化品数量状态、储存包装形 式、周边环境、管理等动态因素相关, 基于危化品动 态风险开展相关安全管理与事故应急处置, 可有效防 范事故致因因素的形成, 将有助于提高危化品全过程 安全管理以及事故应急处置的技术水平。

动态风险的影响因素如图 5 所示。针对危化品灾 害事故在时间、空间、人员、物质、设备、环境以及 管理等方面的动态变化特点, 根据相关动态影响因素 变化规律和概率分布特点预测事故发生概率、后果影 响范围, 实时研判动态风险演化规律。基于动态风险 演化规律研究, 综合构建包含风险辨识-评估-控制一 体化的动态风险管理体系, (如图 6) 以提高事故防 范的技术水平与能力。从全生命周期角度进行风险因 素辨识, 需重点辨识人员、物质、设备、环境等变更 而产生的新的火灾风险因素, 及导致火灾、爆炸事故 引发的连锁风险因素。评估事故发生概率和后果影响 范围大小, 及产生多米诺效应概率。采取替代、隔离 等对策措施提前预控事故风险, 将风险因素的事故后 果和概率最小化。

结合危化品信息监管以及事故应急处置的特点, 应用有关视频、传感器、物联网、信息传输、地理信 息以及事故模拟预测等技术手段, 集危化品信息智能 感知与监控、信息获取与传输, 以及事故预测、应急 处置辅助决策等功能, 将危化品各类信息数据库、区 域应急资源信息数字化、化学品储运企业安全监控与 地理信息有机结合, 开发基于动态风险分析的全生命 周期安全监控与应急辅助决策系统。危化品信息采集 形成应急辅助决策系统及数据库架构如图 7 所示。系 统主要实现危化品储存条件及状态实时监测、显示与 报警, 危化品全生命周期作业定位与在线监管, 危化 品信息数据查询与管理, 危化品事故定量风险评估, 危化品事故发展后果预测, 危化品应急资源管理, 危 化品应急辅助决策以及危化品企业及个人资质管理 等功能, 可为危化品仓储、运输企业及园区消防安全 管理与监管、事故应急救援决策提供支持。

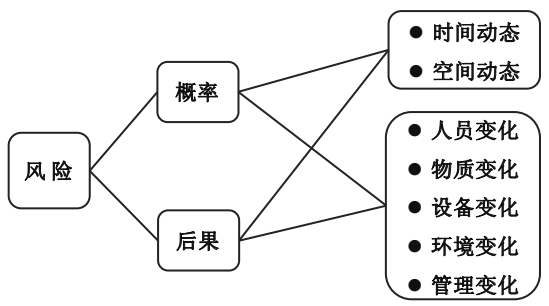

图 5. 动态风险主要影响因素 
Risk Analysis and Crisis Response in Big Data Era (RAC-16)

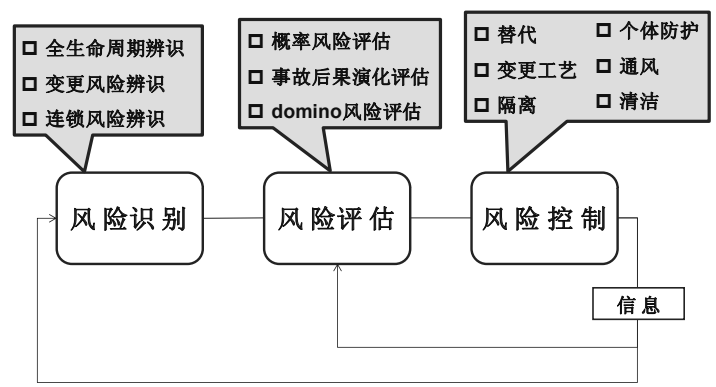

图 6. 动态风险管理体系

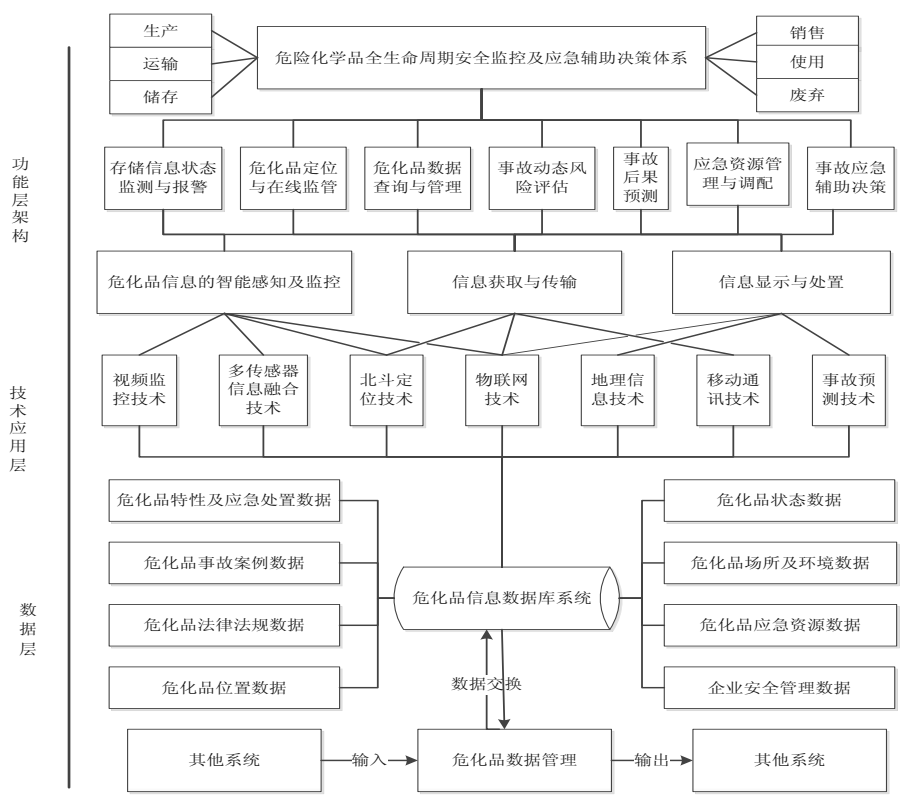

图 7. 危化品全生命周期安全监控及事故应急辅助决策系统架构

\section{6. 结论}

(1) 以 “8.12” 天津瑞海危化品火灾爆炸事故为 典型案例, 绘制事故发展链条并提出 4 个事故防范的 重点关注点。并参考国家相关法律法规, 建立包括 4 类一级致因、14 个二级致因的危化品仓储区燃爆事 故致因因素集。

(2) 采用 DEMATEL 开展因素致因分析, 绘制因 素因果图和网络模型, 笁选事故发生的关键因素。分
析结果表明操作人员失误多为事故发生的本质因素, 其他因素多存在不同程度的反馈和依赖作用。

（3）针针对危化品灾害事故成因及其特点, 以 及危化品风险管理与应急安全管理的技术需求, 构建 了危化品动态风险管理体系, 以及危化品全生命周期 安全监控及事故应急辅助决策体系, 通过加强对危化 品风险管理以及事故应急安全管理, 可有效防范事故 发生、减少事故灾害影响。 
Risk Analysis and Crisis Response in Big Data Era (RAC-16)

\section{Acknowledgements}

This study was supported by Special Foundation Work Intensifying Police with Science and Technology of MPS (No.2014GABJC047), and Key Projects of the Ministry of Public Security Fire Department (No. 2015XFGG02).

\section{致谢}

本研究得到了公安部科技强警基础工作专项 (2014GABJC047) 和公安部消防局重点项目 (2015XFGG02) 的资助。

\section{参考文献}

[1] 柴保身. 我国危险品仓储业发展现状与展望. 物 流工程与管理, 31(12): 19-23, 2009.

[2] 郁振山. 危化品仓库火灾爆炸事故的消防救援. 现代职业安全,09:82-88, 2015.

[3] 桑海泉,康荣学,魏利军. 石油化工码头安全监控 系统研究.中国安全生产科学技术, 4(4): 27-31, 2008.

[4] 孙伟, 董耀华. 基于 RFID 技术的剧毒危化品物 流服务平台研究. 中国安全科学学报, 21(2):147-151, 2011.

[5] 张超，陈晓，陈建国等. 考虑应急救援的危化品 泄露事故后果风险评估. 清华大学学报(自然科 学版), 49(5): 1-5, 2009.

[6] Farid Kadri, Eric Chatelet, Patrick Lallement The Assessment of Risk Caused by Fire and Explosion in Chemical Process Industry: A Domino Effect-Based Study . Journal of Risk Analysis and Crisis Response, 2013, 3(2): 66-76.

[7] Evgeny S. Guryev. Construction of Dynamic Risk Maps for Large Metropolitan Areas. Journal of Risk Analysis and Crisis Response, 2014, 4(2): 72-76.

[8] Fontela E, Gabus A. The DEMATEL Observer Report[R]. DEMATEL 1976 Report.

[9] 天津港“ 8 ・12”特别重大火灾爆炸事故调查报告 [OL]. 中国新闻网, http://www.chinanews.com /sh/2016/02-05/7750596.shtml.

[10] Seyed-Hosseini SM, Safaei N, Asgharpour MJ. Reprioritisation of failures in a system failure mode and effects analysis by deci-sion making trial and evaluation laboratory technique. Reliability Engineering \& System Safety, 91(8): 872 - 881, 2006

[11] 席永涛,陈伟炣,张晓东, 等. 基于 DEMATEL 方 法的海运行为形成因子影响关系分析. 中国安 全科学学报, 04: 14-19, 2012.

[12] 周德群, 章玲. 集成 DEMATEL/ISM 的复杂系统 层次划分研究. 管理科学学报, 11(2):20-26, 2008.
[13] 胡嘉伟,彭伟,薛韦一. 基于 ISM 法的公路隧道火 灾事故致因研究. 中国安全生产科学技术, 10(2):57-62, 2014 